\title{
La fracción parte - todo a través de una mirada gráfica ${ }^{1}$
}

\author{
The fraction part - all through a graphic look
}

A parte fracionária - tudo através de um olhar gráfico

Recibido: mayo de 2013

Aceptado: agosto de 2013
Diana Marcela Acevedo Caro ${ }^{2}$

Miller Alexánder López Miranda ${ }^{3}$

Yeison Andrés Guerrero Osorio ${ }^{4}$

Lina Fernanda Morales Romero $Y^{5}$

\section{Resumen}

El trabajo que se presenta a continuación fue desarrollado durante la clase de investigación en el aula, en el transcurso de ésta, encontramos que aun nosotros como estudiantes universitarios, tenemos problemas al operar entre fracciones, y es por este motivo, que buscamos proponer un método de enseñanza de las mismas, para facilitar el proceso de aprendizaje de los niños con respecto a este tema, aplicando una prueba diagnostico a estudiantes de grado 4, y evidenciando las falencias, que poseen ellos.

Palabras-clave: Matemáticas escolares; números; estructuras numéricas; números racionales; fracción parte-todo; operaciones básicas; otras nociones de Educación Matemática; sistemas de representación gráfica; contexto continuo; contexto discreto.

\section{Abstract}

The work presented here was developed for the kind of research in the classroom, in the course of this, we find that even we as college students, we have problems to operate between fractions, and for this reason, that we propose a teaching method thereof, to facilitate the learning process of children with regard to this issue, applying a diagnostic test students in grade 4, and showing the flaws that have them.

Key words: school Mathematics, numbers, numerical structures, rational numbers, part-whole fraction; basic operations, other notions of Mathematics Education; graphical representation systems, continuous context; discrete context.

Artículo de Investigación.

2 Universidad Distrital Francisco José de Caldas. Bogotá, Colombia. Contacto: dianaacevedo0715@hotmail.com

3 Universidad Distrital Francisco José de Caldas. Bogotá, Colombia. Contacto: millers_18@hotmail.com

4 Universidad Distrital Francisco José de Caldas. Bogotá, Colombia. Contacto: yeisondigital@hotmail.com

5 Universidad Distrital Francisco José de Caldas. Bogotá, Colombia. Contacto: linitam.94@hotmail.com 


\section{Resumo}

O trabalho aqui apresentado foi desenvolvido para o tipo de pesquisa em sala de aula, no curso deste, descobrimos que até mesmo nós, como estudantes universitários, temos problemas para operar entre frações, e por esta razão, que nos propomos um método de ensino da mesma, a fim de facilitar o processo de aprendizagem das crianças com relação a esta questão, a aplicação de um teste de diagnóstico estudantes no grau 4, e mostrando as falhas que elas têm.

Palavras-chave: Matemática da escola, números, estruturas numéricas, números racionais, fração parte-todo, operações básicas, outras noções de Educação Matemática, sistemas de representação gráfica, contexto contínuo; contexto discreto.

\section{Presentación del problema}

Durante la clase de Investigación en el aula I, en el periodo II de 2011, se realizó una prueba individual, al socializar las respuestas de los ejercicios, observamos que gran parte del grupo presentaba falencias al interpretar las fracciones de forma gráfica, Por ejemplo:

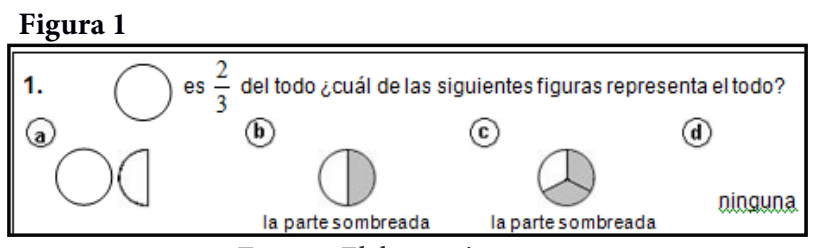

Fuente: Elaboración propia

La figura 1 fue una de las preguntas que debíamos responder, en el momento de socializar sobre las diferentes interpretaciones que cada uno había dado, muchos marcamos la d (ninguna); y el argumento era que no podrían ser las anteriores $(a, b, c)$, puesto que en la opción "a" teníamos $1+$, en la $b$, y en la $c$, sin tener en cuenta que el círculo es la representación de del todo. A lo cual en este momento de la actividad, se tuvo un análisis erróneo de la interpretación gráfica de las fracciones ya que: $\mathrm{Si} \bigcirc$ es igual a , tenemos que $\bigcirc$ = y al tener $\bigcirc+\bigcirc$ obtendríamos ó 1 . Por lo tanto la respuesta es la a.

En los ejercicios posteriores, se encontraron otro tipo de interpretaciones de las gráficas (relacionadas con fracciones como parte todo), lo cual nos llevo a pensar qué: Si en algún momento de nuestra educación básica, a todos de una u otra forma nos enseñan fracciones, ¿Por qué encontramos falencias en el momento de interpretarlas gráficamente?

Se infiere que las fracciones no se nos enseñaron de una manera gráfica por lo tanto no se tenían claros aspectos como: -Existen representaciones gráficas en contextos continuos y discretos, -el todo de una fracción se puede representar como una unidad o un conjunto de cosas, -se pueden operar las fracciones de forma gráfica.

Decidimos elaborar un proyecto de investigación que sea desarrollado con niños de primaria, y más específicamente con niños de tercero, ya que según Pinzón \& Cortés (2005) es en este grado donde el niño empieza a identificar las fracciones y a operar (sumar y restar) fracciones homogéneas, pero como estas son las competencias mínimas, queremos lograr que los niños se apropien también del concepto de fracciones heterogéneas y operen con éstas. Partiendo de esto, surge nuestra pregunta de investigación ¿Cómo enseñar a sumar y restar fracciones parte todo, de forma gráfica, en contextos discretos o continuos?

\section{Marco de referencia conceptual}

Según los lineamientos curriculares y los estándares básicos de competencias en matemáticas, establecidos por el MEN (1998), en Colombia la 
matemática escolar se organiza en cinco clases de pensamiento, los cuales se llevan a cabo con cinco procesos el más pertinente para nosotros es el "pensamiento numérico y los sistemas numéricos". El pensamiento numérico y los sistemas numéricos plantean la organización de actividades que tengan como finalidad la comprensión de la buena utilización del significado de los números y de la numeración; además de esto la comprensión de las operaciones, de las relaciones entre números, y el desarrollo de técnicas de cálculo y estimación.

El razonamiento matemático tiene mucho que ver con las matemáticas como comunicación, modelación y como procedimiento. Razonar en matemáticas según López \& Oliden (2002) tiene que ver con

- Dar cuenta del cómo y del porqué de los procesos que se siguen para llegar a conclusiones.

- Justificarlasestrategiasylos procedimientos puestos en acción en el tratamiento de problemas.

- Formular hipótesis, hacer conjeturas y predicciones, encontrar contraejemplos, usar hechos conocidos, propiedades y relaciones para explicar otros hechos.

- Encontrar patrones y expresarlos matemáticamente.

- Utilizar argumentos propios para exponer ideas, comprendiendo que las matemáticas más que una memorización de reglas y algoritmos, son lógicas y potencian la capacidad de pensar.

Según Fandiño (2005) cuando hablamos de fracciones, nos referimos a las partes de algo (un todo), este término no es reciente y el uso de ellas tampoco, se dice que las fracciones nacen en la civilización egipcia y varias civilizaciones posteriores también utilizaron fracciones como los sumerios, asirios, babilonios, griegos, chinos, hindúes y árabes.

Para plantear la parte didáctica es importante tener en cuenta a que hacemos referencia al hablar de didáctica.
En todo proceso de enseñanza - aprendizaje es necesario que haya didáctica, una de las concepciones de la didáctica, la cual representa el arte de enseñar, es decir es un conjunto de medios para que alguna ciencia sea enseñada de la mejor manera.

\section{Metodología}

Buscamos una propuesta diferente a la tradicional, que nos permita enseñarles a los estudiantes de grado tercero, a interpretar y realizar operaciones (suma y resta) con fracciones.

Teniendo en cuenta que la investigación acción según Ebbutt \& Elliot (1985) está definida como "El estudio sistemático de tentativas de cambio y mejoras educativas, realizadas por los maestros a través de sus propias prácticas y por medio de la reflexión sobre los efectos de su acción"

Primero se hizo el reconocimiento de los estudiantes, luego se la prueba diagnóstico a los alumnos, para saber cuáles son sus conocimientos frente al tema delas fracciones y percibir las falencias en éstas. Teniendo ya la prueba solucionada, se pasó a evaluarla con unos criterios ya establecidos. Con los resultados obtenidos con el análisis de la prueba, pasaremos a realizar la propuesta según las falencias halladas en el grupo evaluado.

\section{Análisis de datos}

La prueba diagnostico fue aplicada a los estudiantes de grado $4^{\circ}$ de primaria (puesto que estos estudiantes ya han visto todas las temáticas de grado tercero), del colegio Liceo Bet-el (cristiano), ubicado en la localidad de suba (Bogotá). Contábamos con la presencia de 14 estudiantes, de ellos 9 eran niños y 5 eran niñas.

Se buscaba analizar era ¿Cuáles son los conocimientos de los niños de grado cuarto $4^{\circ}$ 
del Liceo Bet-el frente al tema de fracciones en contextos discretos o continuos?

Antes de aplicar la prueba, se realizaron algunos ejercicios predestinados para "romper el hielo" luego de saber el nombre y la edad de los niños, pasamos a realizar ejercicios sencillos entre todos.

\section{Conclusiones}

Encontramos que los niños tienen falencias al definir una fracción, la mayoría confunden las fracciones homogéneas con las heterogéneas, aunque se les facilita más interpretar las fracciones propias, que las impropias, y se les dificulta operar (sumar y/o restar) de forma gráfica, principalmente con las fracciones heterogéneas.

En el proceso de enseñanza es muy importante valorar el tipo de pensamiento del educando, para adaptar el tema a desarrollar de una forma más adecuada y comprensible para lograr así un proceso didáctico fructífero en el aprendizaje del alumno.

En las fracciones como parte- todo, la representación gráfica nos da paso a comprender la naturaleza de estas, fortaleciendo la comprensión y la parte inductiva de las operaciones suma y resta entre estas.

\section{Fuente}

Bausela, E. (1992). La docencia a través de la investigación-acción. Revista Iberoamericana de Educación, 20 , 7-36.

Ebbutt,D., \&Elliot,J.(1985). Lainvestigación-acción en educación. Madrid: Ediciones Moratas.

Fandiño, M. (2005). Las fracciones Aspectos conceptuales y didácticos. Colombia: Magisterio.

López, A., \& Oliden, P. (2002). Formulación y validación de un modelo logístico lineal para la tarea de adición y sustracción de fracciones y números mixtos. Red de Revistas Científicas de América Latina, el Caribe, España y Portugal , 802-809.

Pinzón, G., \& Cortés, A. (2005). Estándares Curriculares para Matemáticas Tercer Grado. Colombia: Editorial Hispanoamérica. 


\section{Anexo 1}

\section{Prueba $\mathbf{N}^{\circ} 1$}

Nombre:

Fecha: Grado:

Genero: $\mathrm{F} \_\mathrm{M}$

Para solucionar la siguiente prueba, es necesario desarrollarla en orden, si no entiende algún punto, por favor levantar la mano, es una prueba individual.

1. ¿Para ti qué es una fracción?

2. ¿Cuáles son las fracciones homogéneas y cuáles son las heterogéneas? Da ejemplos.

3. Representa gráficamente las siguientes fracciones

$$
-; \quad-; \quad-; \quad-\text {. }
$$

4. ¿A qué fracción corresponden los siguientes gráficos? Teniendo en cuenta que lo tomado es la parte sombreada.
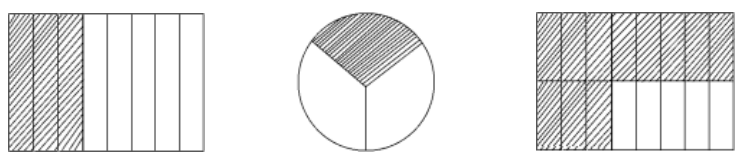

5. Resuelve las siguientes operaciones, mediante los gráficos.

A
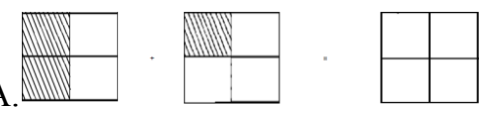

B.

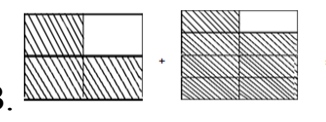

C.
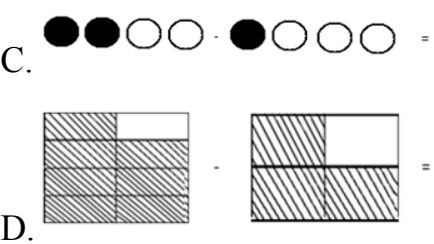

Fuente: Elaboración propia 